EDITORIAL

\title{
Underuse of revascularisation in acute coronary syndromes
}

\author{
Franz-Josef Neumann, Heinz Joachim Büttner
}

\begin{abstract}
Is coronary revascularisation being underused in patients at highest risk, and overused in those at lower risk?
\end{abstract}

Heart 2007;93:147-148. doi: 10.1136/hrt.2006.092643

have more advanced coronary disease. Hence, with increasing GRACE risk score, the proportion of patients with an accepted indication for bypass surgery-as opposed to PCI-will increase. Surgeons, physicians and patients alike may be reluctant to take this choice in the presence of myocardial infarction, advanced age or other high risk features. Supporting this concern, the proportion of patients undergoing bypass surgery as revascularisation modality increased with increasing risk score $(10 \%, 14 \%, 16 \%)$, but this trend could not compensate for the substantial decrease in the use of PCI. As a third point the possibility has to be considered that the proportion of patients with a coronary anatomy not amenable to any form of revascularisation, or with co-morbidities that preclude a reasonable benefit from revascularisation, might increase with increasing GRACE risk score.

\section{IS REVASCULARISATION UNDERUSED IN HIGH-RISK PATIENTS?}

Because of the inherent limitations of registries and the limited number of variables that can be gathered in large cohorts, such as GRACE, the relative contribution of each of the potential mechanisms for the inverse relation between the frequency of revascularisation and the GRACE risk score cannot be assessed with certainty. The most important question-and one that should affect practice patterns-is whether there was underuse of revascularisation in high-risk situations and overuse with low-risk patients. The comparison of high-volume and low-volume hospitals reported by Fox and co-workers may give some clue in this respect.

In the highest tertile of the GRACE risk score, the use of revascularisation varied considerably between tertiles of hospitals stratified according to the percentage of patients with acute coronary syndromes undergoing coronary angiographyfrom $18 \%$ in the lower tertile to $60 \%$ in the upper tertile. Assuming comparability in patient characteristics, this variability demonstrates that the hospitals of the lower tertiles of resource utilisation fell short of the potentials of revascularisation. The question remains as to whether there was still underuse of revascularisation in the high-end hospitals. In this respect, it is noteworthy that in the most recent study including high-risk acute coronary syndromes, ICTUS, ${ }^{4}$ the percentage of patients undergoing revascularisation during the initial hospitalisation was $76 \%$ in the invasive arm Franz-Josef Neumann, MD, Herz-Zentrum Bad Krozingen, Südring 15, 79189 Bad Krozingen, Germany; franz-josef. neumann@herzzentrum.de

See end of article for authors' affiliations

.....................

.......................... $\mathrm{n}$ acute coronary syndromes with high-risk
features, expeditious coronary revascularisation is the treatment of choice. Irrespective of the time from onset of pain, ${ }^{1}$ direct percutaneous coronary intervention (PCI) is the best treatment unless it causes a delay of more than two hours compared with fibrinolysis. ${ }^{3}$ Although challenged
by the recent ICTUS (Invasive versus Conservative Treatment in Unstable Coronary Syndromes) trial, larization in non-ST elevation acute coronary syndromes irrespective of the primary success of
medical treatment. ${ }^{5-7}$ The high-risk features that GRACE REGISTRY (trategies is patients who are at the highest risk and, he inl obtain the largest benefit. In a study repor such strategy was pursued in the hospitals with cath-lab facilities participating in GRACE. To their substantially with increasing risk. In the tertil pre GRACE risk score, the proportion tertile, and $35 \%$ in the highest risk tertile.

There are several potential explanations for this finding. The most obvious explanation may be circumscribed with "picking the low hanging fruit". In other words, interventional cardiologists may have a low threshold for treating easy lesions, predominantly found in the lower risk patients, and tend to leave the more complicated lesions, predominantly found in higher risk patients, for medical treatment. But there are other explanations that also need attention. In view of the large age differences between tertiles of the GRACE risk score ( 55 years, 66 years and 76 years), it is conceivable that patients of the upper tertiles will

Abbreviations: GRACE, Global Registry of Acute Coronary Events; ICTUS, Invasive versus Conservative Treatment in Unstable Coronary Syndromes; $\mathrm{PCl}$, percutaneous coronary intervention 
and $40 \%$ in the conservative arm without any significant difference in primary outcome. Likewise, in ISAR-COOL, a study on high risk acute coronary syndromes, the in-hospital revascularisation rate was $75 \%{ }^{10}$ To our experience, these rates from dedicated randomised studies are comparable to the revascularisation rates in unselected high-risk cohorts of patients with acute coronary syndromes treated at specialised high-volume interventional centres. ${ }^{11}$ Currently available data, therefore, indicate that a sizable proportion of patients with acute coronary syndromes and high risk features cannot be considered candidates for coronary revascularisation. Nevertheless, these comparisons do not exclude that the rate of revascularisation in the high-end hospitals of GRACE was still suboptimal. Reassuringly, we may, however, assume that they did not miss the optimum to a large degree.

\section{OVERUSE OF PCI}

Another concern is the overuse of PCI in patients with low GRACE risk scores, particularly in the hospitals of GRACE in the highest tertiles of coronary angiography. In these patients, PCI rates reached $78 \%$ and total revascularisation rates $84 \%$. In interpreting these findings, it has to be considered that GRACE specifically selected a high risk cohort. Hence, in a widely accepted point of view, the patient population regarded as "low-risk" in the current analysis of GRACE could well be regarded as having a rather high risk. ${ }^{57}$ This is because patients had to present with electrocardiographic changes or positive myocardial marker proteins to be included in GRACE. ${ }^{9}$ FRISC-2 and TACTICS demonstrated benefit from revascularisation in patients with ST segment changes or positive myocardial marker proteins. ${ }^{12}{ }^{13}$ Hence, the intervention-even in the lower risk spectrum of GRACE-was clinically indicated for improvement of outcome and endorsed by current European guidelines. ${ }^{5}$

Important messages can be derived from the current analysis of GRACE. As the authors point out, their study demonstrates that, particularly where resources are limited, ascertainment of risk has too little impact on patient allocation to revascularisation therapy. In our view, it is an important additional message that patients are more likely to receive appropriate revascularisation therapy when transferred to hospitals with adequate technical resources, staffing and expertise. Not all hospitals with cath-lab facilities can provide the full spectrum of the latest interventional and surgical techniques. Based on risk ascertainment, the threshold for transferring patients to specialised referral centres when needed should be low.

\section{Authors' affiliations}

F-J Neumann, H J Büttner, Herz-Zentrum Bad Krozingen, Bad Krozingen, Germany

\section{REFERENCES}

1 Boersma E, for The Primary Coronary Angioplasty vs. Thrombolysis Group. Does time matter? A pooled analysis of randomized clinical trials comparing primary percutaneous coronary intervention and in-hospital fibrinolysis in acute myocardial infarction patients. Eur Heart J 2006;27:779-88.

2 Keeley EC, Boura JA, Grines CL. Primary angioplasty versus intravenous thrombolytic therapy for acute myocardial infarction: a quantitative review of 23 randomised trials. Lancet 2003;361:13-20.

3 Betriu A. Masotti M. Comparison of mortality rates in acute myocardial infarction treated by percutaneous coronary intervention versus fibrinolysis. Am J Cardiol 2005;95:100-1.

4 de Winter RJ, Windhausen $\mathrm{F}$, Cornel JH, et al, for the Invasive versus Conservative Treatment in Unstable Coronary Syndromes (ICTUS) Investigators. Early invasive versus selectively invasive management for acute coronary syndromes. N Engl J Med 2005;353:1095-104.

5 Bertrand ME, Simoons ML, Fox KA, et al. Management of acute coronary syndromes in patients presenting without persistent ST-segment elevation. Eur Heart J 2002;23:1809-40.

6 Mehta SR, Cannon CP, Fox KA, et al. Routine vs selective invasive strategies in patients with acute coronary syndromes: a collaborative meta-analysis of randomized trials. JAMA 2005;293:2908-17.

7 Silber S, Albertsson P, Aviles FF, et al. Guidelines for percutaneous coronary interventions. Eur Heart J 2005;26:804-47.

8 Collinson J, Flather MD, Fox KA, et al. Clinical outcomes, risk stratification and practice patterns of unstable angina and myocardial infarction without ST elevation: Prospective Registry of Acute Ischaemic Syndromes in the UK (PRAISUK). Eur Heart J 2000;21:1450-7.

9 Fox KAA, Anderson FA, Dabbous $\mathrm{OH}$, et al. Intervention in acute coronary syndromes: do patients undergo intervention on the basis of their risk characteristics? The Global Registry of Acute Coronary Events (GRACE). Heart 2007;93:177-82

10 Neumann FJ, Kastrati A, Pogatsa-Murray G, et al. Evaluation of prolonged antithrombotic pretreatment ("cooling-off" strategy) before intervention in patients with unstable coronary syndromes: a randomized controlled trial. JAMA 2003;290:1593-9

11 Mueller C, Buettner HJ, Hodgson JM, et al. Inflammation and long-term mortality after non-ST elevation acute coronary syndrome treated with a very early invasive strategy in 1042 consecutive patients. Circulation 2002;105:1412-5.

12 FRISC II Investigators. Invasive compared with non-invasive treatment in unstable coronary-artery disease: FRISC II prospective randomised multicentre study. Lancet 1999;354:708-15.

13 Cannon CP, Weintraub WS, Demopoulos LA, et al. Comparison of early invasive and conservative strategies in patients with unstable coronary syndromes treated with the glycoprotein Ilb/llla inhibitor tirofiban. N Engl J Med $2001 ; 344: 1879-87$ 